\title{
Altered response to risky decisions and reward in patients with obsessive-compulsive disorder
}

\author{
Pedro Silva Moreira, MSc, PhD; Julian Macoveanu, MSc, PhD; Paulo Marques, MEng, PhD; \\ Ana Coelho, MEng; Ricardo Magalhães, MEng, PhD; Hartwig R. Siebner, MD, DMSc; \\ José Miguel Soares, MEng, PhD; Nuno Sousa, MD, PhD; Pedro Morgado, MD, PhD
}

\begin{abstract}
Background: Patients with obsessive-compulsive disorder (OCD) employ ritualistic behaviours to reduce or even neutralize the anxiety provoked by their obsessions. The presence of excessive rumination and indecision has motivated the view of OCD as a disorder of decision-making. Most studies have focused on the "cold," cognitive aspects of decision-making. This study expands current understanding of OCD by characterizing the abnormalities associated with affective, or "hot" decision-making. Methods: We performed a functional MRI study in a sample of 34 patients with OCD and 33 sex- and age-matched healthy controls, during which participants made 2-choice gambles taking varying levels of risk. Results: During risky decisions, patients showed significantly reduced task-related activation in the posterior cingulum, lingual gyrus and anterior cingulate cortex. We identified significant group $\times$ risk interactions in the calcarine cortex, precuneus, amygdala and anterior cingulate cortex. During the outcome phase, patients with OCD showed stronger activation of the orbitofrontal cortex, anterior cingulate cortex and putamen in response to unexpected losses. Limitations: The group of patients not receiving medication was very small $(n=5)$, which precluded us from assessing the effect of medication on risk-taking behaviour in these patients. Conclusion: Obsessive-compulsive disorder is associated with abnormal brain activity patterns during risky decision-making in a set of brain regions that have been consistently implicated in the processing of reward prediction errors. Alterations in affective "hot" processes implicated in decision-making may contribute to increased indecisiveness and intolerance to uncertainty in patients with OCD.
\end{abstract}

\section{Introduction}

Obsessive-compulsive disorder (OCD) is a psychiatric disorder characterized by intrusive and repetitive thoughts that cause extreme levels of anxiety (obsessions), and by repetitive behaviours or mental acts that are repeated in ritualistic, stereotyped succession (compulsions). ${ }^{1,2}$ Although patients with OCD are aware of the exaggerated nature of their ritualistic acts, these behaviours allow them to reduce or neutralize the anxiety and the negative affect associated with the obsessions. ${ }^{2}$ As a consequence, these behavioural patterns are continuously reinforced and become established as natural rewards. ${ }^{3}$ Depending on the severity of the disorder, such behaviours may persist for hours, explained by the fact that the patient keeps facing the "what if?" question (i.e., the fear that something bad may happen if they do not enact the ritual). ${ }^{2}$ This uncertainty-related activity becomes generalized, so that people with OCD become highly indecisive when choosing between simple alternatives in many real-life situations. ${ }^{4}$ Patients with OCD frequently engage in pathological rumi- nation and doubt about whether a particular choice was properly made. Such behaviour has led to the conceptualization of OCD as a disorder of decision-making. ${ }^{5}$ Several studies have, in fact, demonstrated that patients with OCD display behavioural and neurobiological alterations during decision-making in laboratory settings. ${ }^{6,7}$ Previous reports also have demonstrated that the specific nature of the behavioural task may selectively affect patients' performance. When the task is characterized by explicit, stable rules, such as the Iowa Gambling Task, ${ }^{8}$ patients display a behavioural profile similar to that of healthy individuals. On the other hand, when the decision-making task has implicit rules, such as the Game of Dice Task, the performance of patients with OCD is very different from that of controls. ${ }^{9}$

A useful approach to better understanding the mechanisms of impaired decision-making in OCD is to examine the fundamental neurobiological attributes of decision-making in these patients. Abnormal functioning in cortical-striatalthalamic-cortical loops have been continuously proposed as a critical pathway for the manifestation and progression of

Correspondence to: P.S. Moreira, Life and Health Sciences Research Institute (ICVS), School of Medicine, University of Minho, Campus Gualtar, 4710-057 Braga, Portugal; pedromsmoreira@ gmail.com

Submitted Nov. 16, 2018; Revised Mar. 27, 2019; Accepted Apr. 29, 2019; Published online Sept. 11, 2019

DOI: 10.1503/jpn.180226 
OCD symptoms, during rest and during symptom provocation. ${ }^{6,10,11}$ Evidence from meta-analyses has implicated several components of these loops as critical hubs for decision-making. ${ }^{12}$ In fact, there is some evidence of desynchronization between frontolimbic and frontostriatal regions during decision-making in patients with OCD. ${ }^{5,13}$ As a consequence, it has been suggested that patients with OCD may experience abnormal processing of reward history and choice valuation: in other words, altered homeostatic processing of decision-making. ${ }^{13}$

Despite the growing literature exploring the neurobiological underpinnings of decision-making in patients with OCD, most studies have used tasks that emphasize the cognitive or "cool," deliberative component of decision-making, as seen in choice involving ambiguity. ${ }^{12}$ In contrast, the study of "hot," affective processing, involving choices associated with risk, ${ }^{14}$ has been relatively underexplored. For this reason, we aimed to investigate risky decision-making patterns in patients with OCD. To do so, using functional MRI, we evaluated the performance of patients with OCD and sexand age-matched healthy controls with a forced-choice gambling task. Our goal was to characterize risky decisionmaking behaviour in patients with OCD and to quantify the regional neural responses associated with decision and feedback at varying levels of risk.

\section{Methods}

\section{Participants}

Sixty-seven right-handed people (34 patients with OCD and 33 healthy controls) without a previous history of neurologic disorders participated in this study. Diagnosis of OCD was established using a semi-structured interview based on DSM-IV-TR and the Yale-Brown Obsessive-Compulsive Scale (Y-BOCS) ${ }^{15}$ Patients with OCD were also evaluated for symptoms of anxiety and depression using the Hamilton Anxiety Rating Scale (HAM-A) and the Hamilton Depression Rating Scale (HAM-D), ${ }^{16}$ respectively. Almost all patients with OCD were taking medication $(87.9 \%)$, primarily a selective serotonin reuptake inhibitor (fluoxetine, fluvoxamine or setraline).

The study was conducted according to the principles of the Declaration of Helsinki and was approved by the Ethics Committee of Hospital de Braga (Portugal). The study goals were explained to participants, and written informed consent was obtained.

\section{Gambling task}

During the fMRI session, participants performed a card gambling task adapted from Macoveanu and colleagues. ${ }^{17}$ Before to each trial, participants were informed of the accumulated (hypothetical) reward (information phase; duration $=1.1 \mathrm{~s}$ ) and the value of the upcoming bet. Next, participants were presented with a screen displaying 7 cards face down, randomly distributed into 2 decks (decision phase; variable duration between $3.5 \mathrm{~s}$ and $6 \mathrm{~s}$ ) and were informed that the "ace of hearts" was hidden in one of the decks. Participants then had to guess the deck containing this card. After this, participants were presented with feedback, in which they could receive a reward or lose the bet (feedback phase; duration $500 \mathrm{~ms}$ ). The task comprised distinct risk levels, with a parametric variation of the odds (range of probability $1 / 7$ to $6 / 7$; Appendix 1, Figure S1, available at jpn.ca/180226-a1). The task was presented as an even distribution of choices across all risk levels, enabling us to study both risk-seeking and riskavoidance behaviour. In the present study, we focused on analysis of risk-taking behaviour. The experimental task consisted of 2 separate runs. Each run lasted for 11 minutes and comprised 112 trials (28 choices between 1 and 6 cards, 28 choices between 2 and 5 cards, 28 choices between 3 and 4 cards, and 28 null events of the same length as a real event where a fixation cross was presented instead of the task screen).

\section{MRI acquisition}

Imaging was performed using a clinically approved $1.5 \mathrm{~T}$ Magnetom Avanto MRI scanner (Siemens) using a 12-channel receive-only head coil. First, we acquired a structural $T_{1}$-weighted $3 \mathrm{D}$ magnetization prepared rapid gradient echo (MPRAGE) scan using the following parameters: 176 sagittal slices, repetition time $2730 \mathrm{~ms}$, echo time $3.48 \mathrm{~ms}$, slice thickness $1 \mathrm{~mm}$, slice gap $0 \mathrm{~mm}$, voxel size $1 \times$ $1 \times 1 \mathrm{~mm}^{2}$, field of view $256 \times 256 \mathrm{~mm}$, flip angle $7^{\circ}$. For the fMRI scan, we acquired a $T_{2}{ }^{*}$-weighted echo planar imaging (EPI) sequence, sensitive to blood-oxygen level-dependent (BOLD) contrast in 2 consecutive runs (each with 315 volumes, and a 1-minute break between acquisitions). The parameters of the EPI sequence were as follows: 38 slices, repetition time $2500 \mathrm{~ms}$, echo time $30 \mathrm{~ms}$, slice thickness $3 \mathrm{~mm}$, voxel size $3 \times 3 \times 3.6 \mathrm{~mm}$, field of view $256 \times$ $256 \mathrm{~mm}$, flip angle $90^{\circ}$.

\section{MRI processing}

The preprocessing pipeline of the functional scans was implemented using SPM12 (www.fil.ion.ucl.ac.uk/spm/ software/spm12/) and included the following steps: (1) slicetiming correction; (2) realignment of the acquired functional images to the mean to correct for head motion; (3) coregistration of the $T_{1}$-MPRAGE structural image to the mean EPI; (4) normalization of participants' images into Montreal Neurological Institute space; and (5) spatial smoothing with a kernel of $8 \mathrm{~mm}$ (full width at half maximum). All resulting images underwent visual inspection for quality control.

\section{Statistical analysis}

We conducted analysis of the number of responses and reaction time for different risk levels using mixed-design factorial ANOVA models, where group was the between-participants factor and risk level was the within-participants factor. Because the experimental paradigm consisted of a forced-choice design with paired risk levels, only 3 conditions were considered ( $1 / 7$ with $6 / 7,2 / 7$ with $5 / 7$, and $3 / 7$ with $4 / 7$ ) for 
the number of choices, with the goal of avoiding collinearity. For analysis of reaction times, we considered each of the 6 risk levels individually.

We conducted the first-level analysis by modelling 3 regressors for the choice phase (high, medium and low risk levels) and 6 regressors for the outcome phase (negative and positive outcomes for high, medium and low risk levels). We also included 6 nuisance regressors, corresponding to 3 directions of translation and 3 axes of rotation, in the model. Regressors were convolved with the canonical hemodynamic response function.

For the second level analysis, we implemented voxel-wise analyses with GLM Flex (http://mrtools.mgh.harvard.edu/ index.php/Main_Page), using mixed-design ANOVA models, with 1 within-participants factor (risk level) and 1 betweenparticipants factor (group). We included the results from the first-level analysis to estimate within- and between-group effects. We implemented 2 distinct approaches to detect statistically significant differences: a fully exploratory model, in which no theoretical regions of interest (ROIs) were defined; and a ROI-driven approach. In the case of the former, we performed 10000 Monte Carlo simulations using 3dFWHMx (estimating intrinsic smoothness based on residuals) and 3dClustSim (estimating probability of false positives), together with the autocorrelation function (-acf), from the AFNI software suite (https://afni.nimh.nih.gov). This approach provided an accurate estimation of cluster size, controlled for family-wise error and accounted for spherical nonGaussian spatial autocorrelation of the fMRI signal. Results from these simulations indicated that for voxel-wise intensity thresholds of $p<0.001$ and $p<0.005$ (restricted to the grouplevel brain mask), cluster extent thresholds of 110 and 250 contiguous voxels (first- and second-intensity thresholds, respectively) would be necessary to achieve an overall type I error rate of $p<0.05$ (corrected for multiple comparisons). For the second approach, and following the strategy described in previous reports, we conducted ROI analyses on brain regions that were shown to be associated with the processing of expected value and reward in a large-scale metaanalysis of neuroimaging studies, ${ }^{18}$ including striatal nuclei (including the caudate, putamen and nucleus accumbens), the insula, the orbitofrontal cortex, the anterior cingulate cortex (ACC) and the amygdala. All ROIs were anatomically defined based on the Automated Anatomic Labelling atlas. ${ }^{19}$ For the ROI-based approach, we created a mask including the above-mentioned ROIs. We defined an arbitrary uncorrected $p$ value of $p<0.005$, with a minimum cluster extension of 10 contiguous voxels, as the threshold for statistical significance. We recognize that this approach is likely to increase the false positive rate, which raises the need to exercise caution in interpretation of these results (which should be made in relation to previous findings). It is also the case that similar strategies have been implemented to deal with the small size of a priori structures, such as the nucleus accumbens. ${ }^{20-22}$

We performed post hoc analyses to compare the BOLD signal between risk conditions and for the decomposition of interaction effects. We extracted the mean BOLD signal for each (whole-brain and ROI levels) result. We then imple- mented correlation analyses to assess the effect of clinical parameters (duration of illness and Y-BOCS, HAM-A and HAM-D total scores) on behavioural and/or neuroimaging findings. We employed both frequentist and Bayesian analyses, the latter being a valuable approach for dealing with multiple comparisons. For the Bayesian analysis, we compared 2 models for each pair-wise association: the null hypothesis model $(\mathrm{H} 0)$, which assumed a bivariate normal distribution with zero covariance, and the alternative hypothesis model $(\mathrm{H} 1)$, which assumed that variables distributed according to a bivariate normal distribution with a nonzero covariance were related..$^{23}$ Bayes factors (BF), the ratios between the marginal likelihoods of the alternative and null models, were interpreted according to Jeffreys ${ }^{24}$ cutoffs: anecdotal (BF10 1 to 3), moderate (BF10 3 to 10), strong (BF10 10 to 30$)$, very strong (BF10 30 to 100$)$ or extreme (BF10 > 100) relative evidence. The association between clinical variables and fMRI results were implemented in JASP (version 0.9.0; https://jasp-stats.org).

\section{Results}

Characteristics of the study sample are described in Table 1.

\section{Behavioural results}

Patients with OCD and healthy controls displayed similar choice profiles across the 6 risk levels (Table 1). Response number was a linear function of risk level: participants of both groups made more choices at lower levels of risk. In addition, we found a significant effect of risk level on reaction times $\left(F_{3.9,224.7}=3.943 ; p<0.001\right)$, which was most fitted by a quadratic function, such that the choices $2 / 7$ and $3 / 7$ were associated with longer response times.

\section{Patterns of brain activity during the decision phase}

We observed significant main effects of risk condition on patterns of brain activity, such that higher risk elicited increased activation of the nucleus accumbens, the anterior insula and the cingulum middle. On the other hand, we noted that the activity of the posterior insula (ROI approach) was significantly diminished in response to high risk (Table 2, Fig. 1). We observed significant effects of group during the choice phase on the activity of the posterior cingulum, the lingual gyrus and the pregenual division of the ACC: patients with OCD displayed decreased activation of these regions during the decision phase, independently of the risk condition (Table 3, Fig. 2A). We found significant group $\times$ risk interactions for the amygdala and the ACC (considering the ROI approach). Post hoc analysis indicated that patients with OCD displayed a larger reduction of the BOLD signal in the amygdala during high-risk choices compared to low-risk choices $\left(t_{33}=-2.16 ; p=\right.$ 0.038 ; $d=-0.371)$. We observed the opposite pattern for healthy controls (i.e., a greater reduction of amygdala activity for low-risk options; $t_{32}=3.09 ; p=0.004 ; d=0.537$ ). The healthy control group was characterized by greater deactivation of the ACC during high-risk choices (compared with low-risk 
Table 1: Sociodemographic and clinical characteristics of patients with $O C D$ and healthy controls*

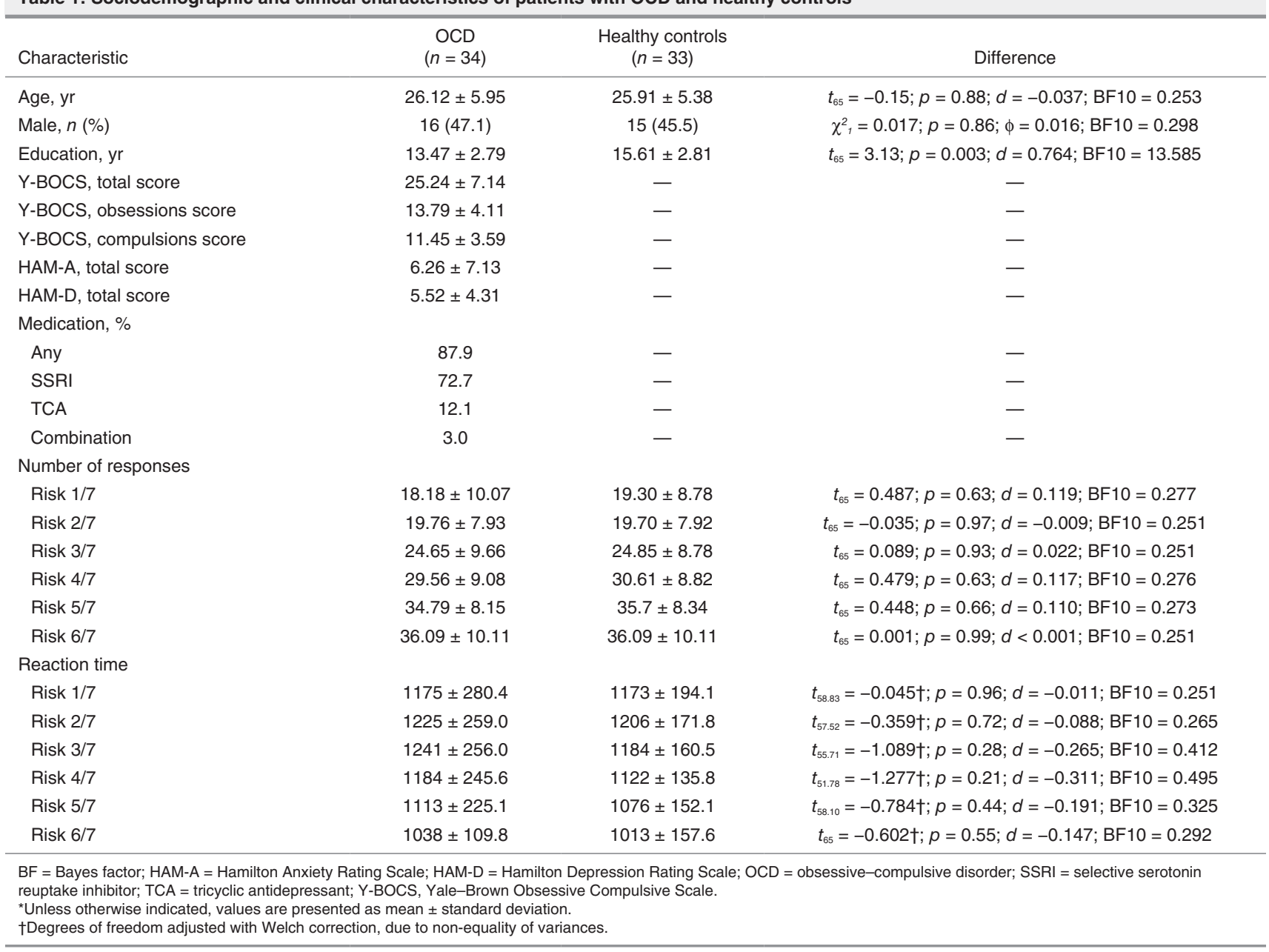

Table 2: Effects of condition during the anticipation phase

\begin{tabular}{cccl}
\hline $\begin{array}{l}\text { Cluster } \\
\text { extension }\end{array}$ & $\begin{array}{c}\text { Peak } \\
\text { intensity }\end{array}$ & MNI, $x, y, z^{*}$ & \multicolumn{1}{c}{ Label } \\
\hline $\begin{array}{c}\text { Effect of risk } \\
p_{\text {FWE }}<0.05\end{array}$ & & & \\
161 & 43.34 & $16,10,-6$ & Right putamen \\
253 & 40.95 & $-12,8,-6$ & Left putamen \\
186 & 30.4 & $-30,22,-8$ & Left insula \\
44 & 26.49 & $12,-64,40$ & Right precuneus \\
211 & 25.89 & $6,36,34$ & $\begin{array}{l}\text { Right cingulum } \\
\text { middle }\end{array}$ \\
23 & 22.37 & $32,20,-6$ & Right insula \\
33 & 21.01 & $-48,-44,56$ & Left inferior parietal \\
11 & 16.19 & $-12,-86,-10$ & Right lingual gyrus \\
$p<0.005$ & & & \\
86 & $9.03 \dagger$ & $42,-10,4$ & Right insula \\
\hline
\end{tabular}

$\mathrm{FWE}=$ family-wise error $\mathrm{MNI}=$ Montreal Neurological Institute; $\mathrm{ROI}=$ region of interest.

${ }^{*}$ Coordinates are presented in the MNI standard space.

* Uncorrected results for ROI-driven approach. choices; $\left.t_{32}=-2.74 ; p=0.010 ; d=-0.478\right)$, and the OCD group demonstrated greater ACC deactivation during low-risk choices $\left(t_{33}=2.58 ; p=0.014 ; d=0.443\right.$; Table 3, Fig. 2B). These between-group and interaction effects remained statistically significant after controlling for education level.

\section{Brain response during the outcome phase}

In the outcome phase, we observed that although there were no significant differences between groups in patterns of brain activity, there were significant effects of condition: both groups showed greater activity in the cerebellum and anterior insula in response to negative outcomes at higher risk levels. Using the ROI-driven approach, we found significant group $\times$ condition interaction effects in the subgenual division of the ACC and right putamen. Specifically, when receiving negative outcomes after low-risk choices, the OCD group manifested considerably greater deactivation of the putamen $\left(t_{65}=3.24 ; p=0.002 ; d=0.792\right)$ and the ACC $\left(t_{65}=2.44 ; p=\right.$ $0.017 ; d=0.597)$ compared to healthy controls. In contrast, the healthy controls group showed larger deactivations of the 


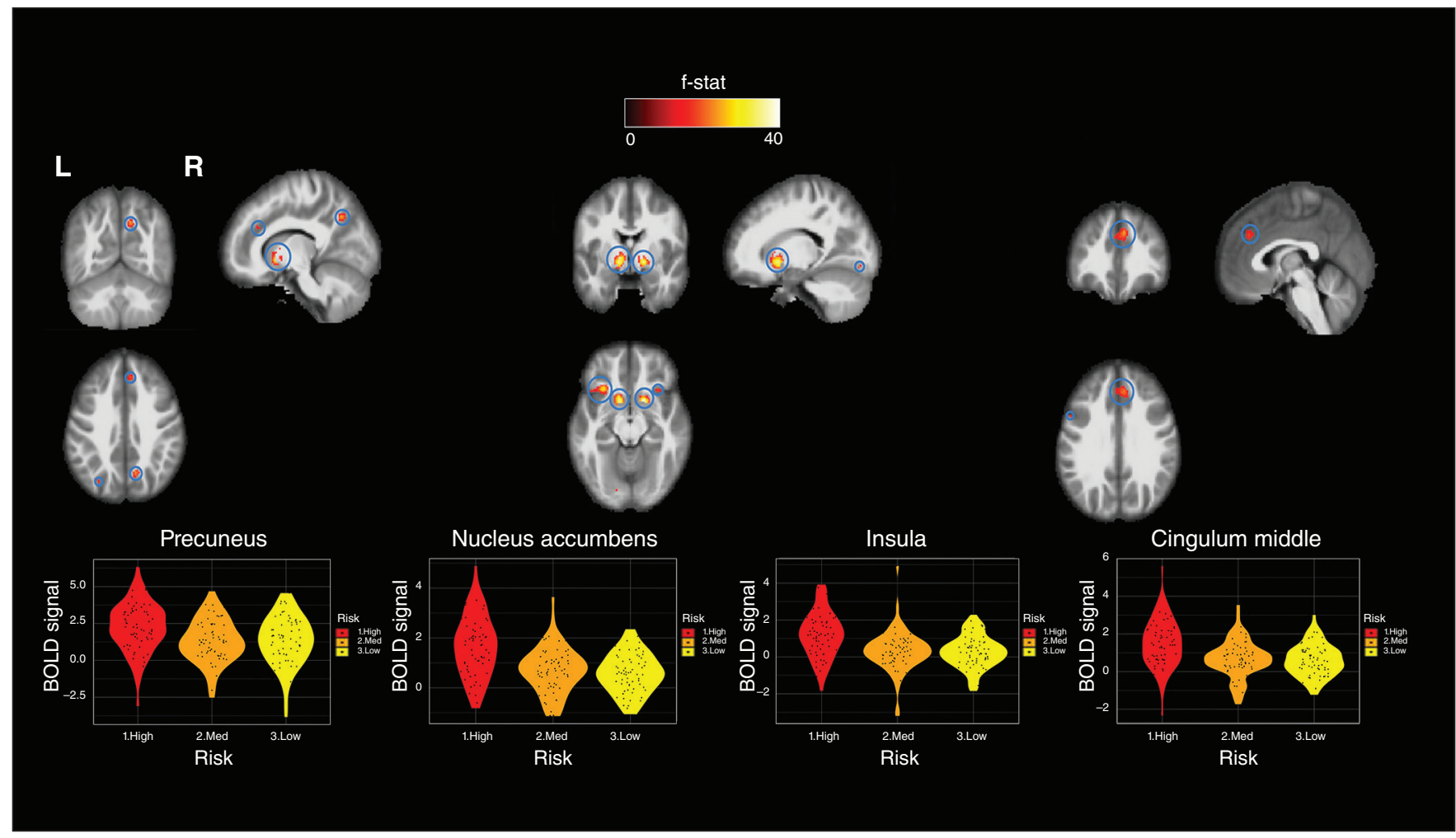

Fig. 1: Main effects of condition on patterns of brain activity during the decision phase. High-risk choices are associated with increased activity in the precuneus, nucleus accumbens, anterior insula and cingulum middle. Low-risk choices are associated with increased activity in the posterior insula. The shapes of the plots represent kernel densities. BOLD = blood-oxygenation level-dependent.

ACC in response to negative outcomes $\left(t_{48.8}=-2.01 ; p=0.050\right.$; $d=-0.488$ ) after high-risk choices (Table 4, Fig. 3). All findings described for the outcome phase were unchanged after controlling for level of education. We observed no significant differences between patients and controls when the choices resulted in a positive outcome.

\section{Association between brain activity and clinical measures}

We sought indications of correlation between patterns of fMRI findings and the clinical severity of the disorder. There was moderate evidence $(\mathrm{BF} 10=9.37)$ for an association between the BOLD signal of the lingual gyrus during high risk (i.e., $1 / 7$ and $2 / 7$ risk levels) in the decision phase and the severity of the disorder (as reflected in total Y-BOCS scores). In a similar fashion, there was a significant association between the activity of the posterior cingulum during high risk with depression scores $(r=0.419 ; p=0.030)$, but the Bayesian analysis provided only anecdotal evidence for such an association $(B F 10=2.25)$. Similarly, we obtained significant uncorrected results, with anecdotal evidence for the alternative hypothesis $(\mathrm{BF} 10=1.68)$, for an association between precuneus activity during the decision period to medium-risk choices ( $3 / 7$ and $4 / 7$ choices) and anxiety scores $(r=0.393 ; p=0.043)$. Finally, we observed a significant association, with anecdotal relative evidence $(\mathrm{BF} 10=1.57)$, between the activity of the putamen in response to low-risk choices during the outcome
Table 3: Effects of group and condition $\times$ group interaction during the anticipation phase

\begin{tabular}{|c|c|c|c|}
\hline $\begin{array}{l}\text { Cluster } \\
\text { extension }\end{array}$ & $\begin{array}{c}\text { Peak } \\
\text { intensity }\end{array}$ & $\mathrm{MNI}, x, y, z^{*}$ & Label \\
\hline \multicolumn{4}{|c|}{$\mathrm{OCD}<$ healthy controls } \\
\hline \multicolumn{4}{|c|}{$p<0.001$} \\
\hline 427 & 5.33 & $20,-64,8$ & Right calcarine \\
\hline \multicolumn{4}{|c|}{$p<0.005$} \\
\hline 1240 & 5.33 & $20,64,8$ & Right calcarine \\
\hline 278 & 3.86 & $0,-32,42$ & Left cingulum middle \\
\hline 159 & $3.6 \dagger$ & $-4,32,28$ & Left cingulum anterior \\
\hline \multicolumn{4}{|c|}{ Condition $\times$ group } \\
\hline \multicolumn{4}{|c|}{$p<0.005$} \\
\hline 18 & $7.66 \dagger$ & $-22,-6,-18$ & Left amygdala \\
\hline 12 & $8.34 \dagger$ & $-4,26,-12$ & $\begin{array}{l}\text { Left superior frontal, } \\
\text { medial orbital }\end{array}$ \\
\hline
\end{tabular}

$\mathrm{MNI}=$ Montreal Neurological Institute; $\mathrm{OCD}=$ obsessive-compulsive disorder; $\mathrm{ROI}=$ region of interest.

${ }^{*}$ Coordinates are presented in the MNI standard space.

†Uncorrected results for ROI-driven approach.

phase and Y-BOCS total score $(r=0.356 ; p=0.042)$. We found no significant association between the neuroimaging findings and the duration of the disorder. A full description of the results of the correlation analyses for the significant (wholebrain and ROI levels) regional brain activity is presented in Appendix 1, Table S1. 
A Group

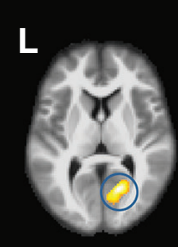

A
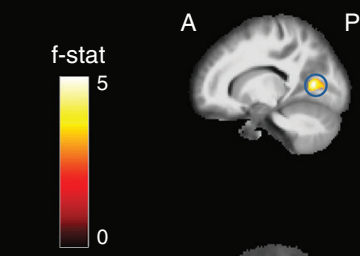

5

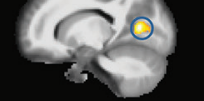

0

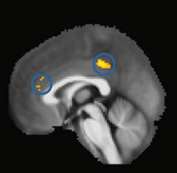

$\mathbf{L}$

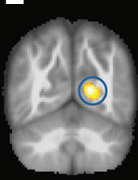

$\mathbf{R}$

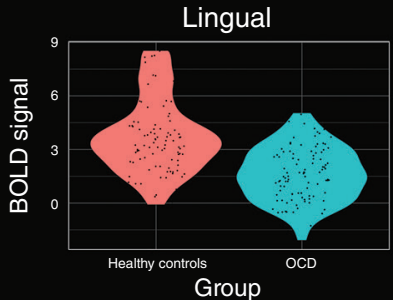

Cingulum
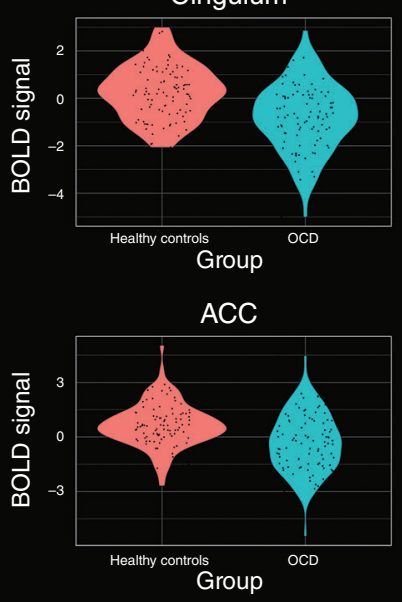

Healthy controls

\section{B Group by condition}

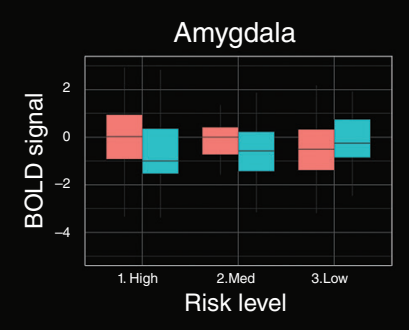

$\mathbf{L}$

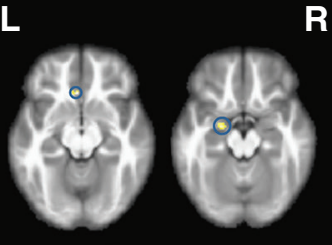

$\mathbf{R}$
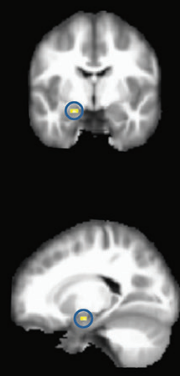

f-stat

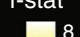

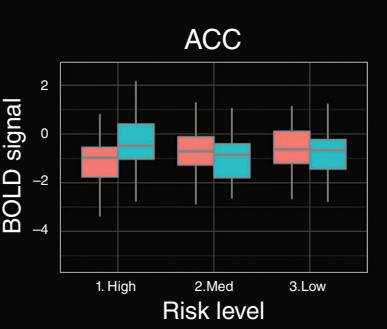

OCD

Fig. 2: (A) Effects of group during the decision phase. Group had a significant effect on the patterns of brain activity of the posterior cingulum, lingual gyrus and ACC. (B) Group $\times$ condition interaction effects during the decision phase on the amygdala and ACC. The shapes of the plots represent kernel densities. ACC = anterior cingulate cortex; BOLD = blood-oxygenation level-dependent.

\section{Discussion}

We conducted an fMRI study designed to examine the neural correlates of risky decision-making in patients with OCD. We observed that patients with the disorder manifested patterns of brain activity during the decision and feedback phases on a gambling task that differed from those of healthy controls. The most pronounced findings were associated with specific clinical characteristics of the disorder, namely the severity of obsessive symptoms and anxiety levels.

At the behavioural level, we identified no between-group differences with respect to decision-making behaviour as a function of risk. Although there is accumulating evidence suggesting that these patients manifest high levels of loss aversion, our results did not corroborate these findings. One possible explanation for the absence of significant differences between our experimental groups may be the specific nature of the task, which requires people to select between contrasting risk choices. Another reasonable explanation relies on the fact that most studies demonstrating significant alterations of decision-making behaviour in patients with OCD were conducted mainly with tasks involving ambiguity, rather than explicit risk.

During the decision phase, patients with OCD displayed demonstrably reduced activity in the posterior and anterior cingulate areas, independent of risk level. The ventral ACC has been shown to be of great relevance for the integration of risk and payoff, and therefore for learning the value of actions. ${ }^{25}$ Abnormal patterns of activity in specific divisions of the ACC have been linked to behavioural impairments in patients with OCD, such as those associated with error processing. ${ }^{26}$ Furthermore, a recent meta-analysis reported that activity in the ventral ACC is significantly reduced during inhibitory control. ${ }^{27}$ The posterior cingulum is one important cluster of the default mode network. It is widely accepted that this network plays an important role in emotion processing and self-referential activity, including introspection. ${ }^{28}$ The involvement of visual areas in the pathophysiology of psychiatric disorders has been receiving increasing 
attention in recent years. Altered functional connectivity between the lingual gyrus and the insular cortex has been reported in autism spectrum disorder ${ }^{29}$ and addiction. ${ }^{30}$ Furthermore, recent neuroimaging studies have reported reduced occipital cortex activity in patients with bipolar disorder during periods of mania or depression, but not during euthymic states. ${ }^{31}$ In accordance with this observation, we found that the lingual gyrus was hyperactivated in the OCD group, and this finding was correlated with the severity of the disorder. This region has been shown to be involved in the processing of emotionally charged content ${ }^{32}$ and the generation of somatic arousal. ${ }^{33}$ In previous work, we identified the lingual gyrus as a critical node of a network shown to have diminished functional connectivity in patients with OCD. ${ }^{34}$

Patients with OCD displayed a substantially larger reduction of the BOLD signal in the amygdala in response to
Table 4: Effects of condition and condition $\times$ group interaction during feedback to losses

\begin{tabular}{cccl}
$\begin{array}{l}\text { Cluster } \\
\text { extension }\end{array}$ & $\begin{array}{c}\text { Peak } \\
\text { intensity }\end{array}$ & MNI, $x, y, z^{*}$ & \multicolumn{1}{c}{ Label } \\
\hline $\begin{array}{c}\text { Effect of risk } \\
p_{\text {FWE }}<0.05\end{array}$ & & & \\
54 & 21.2 & $2,-78,-20$ & Cerebellum, vermis 7 \\
14 & 19.12 & $22,58,20$ & Right superior frontal \\
Condition $\times$ group & & & \\
$p<0.005$ & & & \\
46 & $11.21 \dagger$ & $36,-12,-2$ & $\begin{array}{l}\text { Right putamen } \\
\text { Right superior frontal, } \\
46\end{array}$ \\
& $9.32 \dagger$ & $8,36,-10$ & medial orbital \\
\hline
\end{tabular}

$\mathrm{MNI}=$ Montreal Neurological Institute; $\mathrm{ROI}=$ region of interest.

${ }^{*}$ Coordinates are presented in the MNI standard space.

†Uncorrected results for ROI-driven approach.

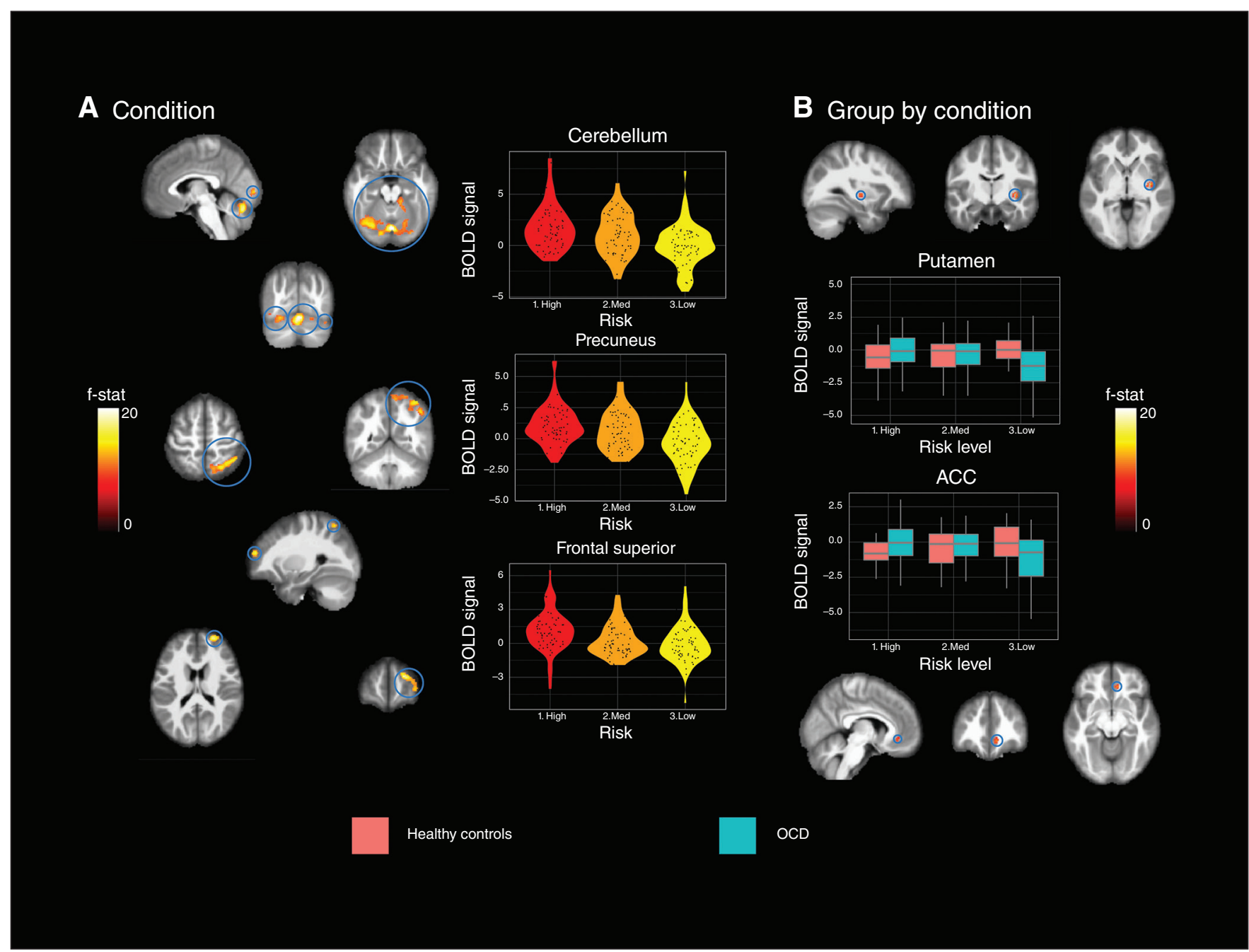

Fig. 3: (A) Condition effects during the feedback to losses. High-risk choices are characterized by significantly increased activity of the cerebellum, precuneus and superior frontal region. (B) Significant group $\times$ risk interactions for the ACC and putamen. For these regions, we found a reduction of the BOLD signal for high-risk choices in the healthy control group. In contrast, we found a reduction of the BOLD signal for low-risk choices in the OCD group. The shapes of the plots represent kernel densities. $A C C=$ anterior cingulate cortex; BOLD = blood-oxygenation level-dependent; OCD = obsessive-compulsive disorder. 
high-risk choices than healthy controls. Abnormal amygdala activation accompanying the threatening anticipation of punishment during risky decision-making in patients with OCD has been previously reported. ${ }^{35}$ Furthermore, it has been demonstrated that increased activation of the amygdala in these patients is directly correlated with the perceived level of uncertainty. ${ }^{36}$ Patients with OCD manifest abnormal emotional processing, marked by a decreased ability to regulate emotions $^{37}$ and a negative bias toward emotional stimuli, in the sense that such stimuli are viewed as more unpleasant and less controllable. ${ }^{38} \mathrm{~A}$ recent meta-analysis revealed that patients with OCD are characterized by altered amygdala responses during emotion processing. ${ }^{39}$ It is important to emphasize that the above-mentioned literature reported increased activation of the amygdala in patients with OCD, in contrast to the decreased activity we have reported. Nevertheless, deactivation of the amygdala is thought to be associated with the cognitive regulation of emotion. ${ }^{40}$ Along with this observation, we hypothesize that patients with OCD may regulate their emotional responses when making high-risk choices. Previous studies have suggested that the synchrony between the amygdala and frontal brain areas underlies a limbic interference with cognitive processing in people with OCD ${ }^{41}$ Extending this proposal, it seems reasonable to suggest limbic interaction with other brain areas during risky decision-making behaviour. One might evaluate this hypothesis by assessing patterns of covariance between limbic regions and higherorder processing-related areas (such as the ACC and the orbitofrontal cortex) during risky decision-making.

In the present study, we found a significant effect of risk level during the decision phase upon activity in the anterior insula: higher-risk choice led to increased activity. It has been frequently suggested that the anterior insula is relevant for higherlevel processing ${ }^{42}$ and risk level, ${ }^{43}$ as a consequence of its involvement in learning the negative value of loss-prediction cues. ${ }^{44,45}$ Activation of the posterior insula has also been associated with lower risk levels, suggesting that different divisions of the insula play opposing roles during risk processing. In fact, evidence from a meta-analysis is consistent with the proposal that different divisions of the insula are characterized by dissociated connectivity profiles. ${ }^{46}$ Recently, it has been proposed that during the evaluation of risky gambles, the anterior insula plays a critical role in tracking arousal, magnitude and risk prediction error, while the posterior insula is primarily involved in urge-processing and signalling homeostatic imbalance. ${ }^{47}$ The nucleus accumbens has also been found to be activated during the processing of high-risk choices. The observation is in accordance with the previously reported involvement of this region in value encoding ${ }^{48}$ and loss aversion. ${ }^{49}$

We identified significant group $\times$ condition interactions in the subgenual ACC and putamen during the feedback period. In each of these regions, patients with OCD demonstrated a larger reduction of the BOLD signal during feedback to lowrisk choices, whereas healthy controls displayed a larger reduction for high-risk choices. Stated differently, patients with OCD suppressed the activity of these brain regions when they perceived unexpected losses. This set of brain regions has repeatedly been shown to be involved in goal-directed decision- making, particularly in learning the sequences of stimuli that lead to a reward. ${ }^{50}$ One recent report suggested that patients with OCD are characterized by abnormal reward prediction errors signalling in the ACC and putamen, which was hypothesized to result in increased indecisiveness and intolerance to uncertainty. ${ }^{51}$ Previously reported abnormal activation of the prefrontal cortex in patients with OCD would tend to support such a view. ${ }^{52}$ It thus seems reasonable to suggest that the abnormal functioning of the identified hubs during risky decision-making in OCD constitutes a response to the processing of unexpected losses. In addition to group $\times$ condition interactions, both of our experimental groups were characterized by significant cerebellar BOLD increases in response to losses. To the best of our knowledge, the involvement of the cerebellum in the processing of loss during decision-making tasks has not been extensively explored. Previous lesion studies have demonstrated that cerebellar damage does contribute to altered decision-making behaviour..$^{53,54}$

\section{Limitations}

Our study had a number of limitations. The first is that almost all patients with OCD were receiving medication. It has been amply documented that pharmacological agents affect patterns of brain activity and functional connectivity. In particular, a recent report suggests that treatment with selective serotonin reuptake inhibitors, through their impact on dopaminergic mesolimbic pathways, may affect decisionmaking performance. ${ }^{55}$ It is thus reasonable to recognize that pharmacological treatment may affect the results obtained here. The diagnosis of OCD spans a range of subtypes and involves heterogeneity of behaviour. As a consequence, it would be interesting to investigate differences between subtypes. Indeed, previous studies have suggested that specific subtypes of OCE may be more susceptible to abnormalities of behavioural performance during decision-making tasks. ${ }^{56}$ Grouping the patients with OCD by subtype would have severely limited the statistical power of our analysis. Another relevant consideration pertains to the use of a more relaxed clusterforming threshold of $p<0.005$, which may have resulted in slightly increased risk for false positives. ${ }^{57}$ However, even though this threshold is commonly adopted by exploratory studies, ${ }^{58}$ our findings were interpreted in line with previous reports. Another relevant question involved the characteristics of the risky decision-making task used in this study. Although the task is well-suited for discriminating between opposing levels of risky decision-making, it forces the participants to select between 2 risk alternatives. This attribute limits the ability to discriminate between participants for each decisionmaking scenario and may have affected between-group differences. Future studies could mitigate this potential limitation by allowing the participant to select from multiple risk choices.

\section{Conclusion}

Our study has shown that unique patterns of brain activity accompany affective, or "hot," decision-making in patients with OCD. We hope that these findings will lead to a better 
understanding of the role of affective processes in the decisionmaking behaviour of people with OCD. We suggest that future studies could shed additional light on the interaction between decision-making and affective processing, by assessing patients with OCD using tasks in which the affective component of the decisions has more salience, such as social decision-making.

Acknowledgements: The authors thank Edward Ganz, MD, for his assistance in reviewing the English language of the manuscript.

Affiliations: From the Life and Health Sciences Research Institute (ICVS), School of Medicine, University of Minho, Braga, Portugal (Moreira, Marques, Coelho, Magalhães, Soares, Sousa, Morgado); the ICVS/3Bs, PT Government Associate Laboratory, 4710-057 Braga/ Guimarães, Portugal (Moreira, Marques, Coelho, Magalhães, Soares, Sousa, Morgado); the Clinical Academic Centre, Braga, 4710-057 Braga, Portugal (Moreira, Marques, Coelho, Magalhães, Soares, Sousa, Morgado); the Psychiatric Centre Copenhagen, Copenhagen University Hospital, Rigshospitalet, Blegdamsvej 9, DK-2100 Copenhagen, Denmark (Macoveanu); the Danish Research Centre for Magnetic Resonance, Centre for Functional and Diagnostic Imaging and Research, Copenhagen University Hospital Hvidovre, Kettegård Allé 30, 2650 Hvidovre, Denmark (Macoveanu, Siebner); the Department of Neurology, Copenhagen University Hospital Bispebjerg, Bispebjerg Bakke 23, 2400 København, Denmark (Siebner); and the Institute for Clinical Medicine, Faculty of Medical and Health Sciences, University of Copenhagen, Copenhagen, Denmark (Siebner).

Funding: P. Moreira and R. Magalhães are supported by FCT fellowship grants (PhD-iHES program) with the references PDE/ BDE /113601/2015 and PDE/BDE/113604/2015, respectively. P. Marques was funded by the Fundação Calouste Gulbenkian (Contract grant number P-139977, project "Better mental health during aging based on temporal prediction of individual brain aging trajectories [TEMPO]"). A. Coelho is supported by a scholarship from the project NORTE-08-5639-FSE-000041 (Norte Portugal Regional Operational Programme, NORTE 2020; UMINHO/BD/51/2017). The present work was supported by SwitchBox-FP7-HEALTH-2010 grant 259772-2 and cofinanced by the Portuguese North Regional Operational Program (ON.2 - O Novo Norte) under the National Strategic Reference Framework (QREN), through the European Regional Development Fund (FEDER). H. Siebner holds a 5-year professorship in precision medicine at the Faculty of Health Sciences and Medicine, University of Copenhagen, which is sponsored by the Lundbeck Foundation (grant no. R186-2015-2138).

Competing interests: $\mathrm{H}$. Siebner has received honoraria as a speaker from Sanofi Genzyme and Novartis, as a consultant from Sanofi Genzyme, and as a senior editor (NeuroImage) from Elsevier. He has received royalties as a book editor from Springer. P. Moreira, J. Macoveanu, P. Marques, A. Coelho, R. Magalhães, J. Soares, N. Sousa and P. Morgado declare no competing interests.

Contributors: J. Macoveanu, H. Siebner, J. Soares, N. Sousa and P. Morgado designed the study. P. Moreira, P. Marques, R. Magalhães and J. Soares acquired the data, which P. Moreira, P. Marques, A. Coelho, H. Siebner, N. Sousa and P. Morgado analyzed. P. Moreira, P. Marques and A. Coelho wrote the article, which all authors reviewed. All authors approved the final version to be published and can certify that no other individuals not listed as authors have made substantial contributions to the paper.

\section{References}

1. Abramowitz JS, Taylor S, McKay D. Obsessive-compulsive disorder. Lancet 2009;374:491-9.

2. Graybiel AM, Rauch SL. Toward a neurobiology of obsessivecompulsive disorder. Neuron 2000;28:343-7.
3. Koch K, Reeb TJ, Rus OG, et al. Increased default mode network connectivity in obsessive-compulsive disorder during reward processing. Front Psychiatry 2018;9:254.

4. Tolin DF, Abramowitz JS, Brigidi BD, et al. Intolerance of uncertainty in obsessive-compulsive disorder. J Anxiety Disord 2003;17:233-42.

5. Sachdev PS, Malhi GS. Obsessive-compulsive behaviour: a disorder of decision-making. Aust N Z J Psychiatry 2005;39:757-63.

6. Cavedini P, Gorini A, Bellodi L. Understanding obsessivecompulsive disorder: focus on decision making. Neuropsychol Rev 2006;16:3-15.

7. Cavedini P, Riboldi G, D'Annucci A, et al. Decision-making heterogeneity in obsessive-compulsive disorder: ventromedial prefrontal cortex function predicts different treatment outcomes. Neuropsychologia 2002;40:205-11.

8. Bechara A, Damasio H, Tranel D, Damasio AR. The Iowa Gambling Task and the somatic marker hypothesis: some questions and answers. Trends Cogn Sci 2005;9:159-62.

9. Starcke K, Tuschen-Caffier B, Markowitsch HJ, et al. Dissociation of decisions in ambiguous and risky situations in obsessivecompulsive disorder. Psychiatry Res 2010;175:114-20.

10. Saxena S, Brody AL, Schwartz JM, et al. Neuroimaging and frontal-subcortical circuitry in obsessive-compulsive disorder. $\mathrm{Br} J$ Psychiatry 1998.

11. Ting JT, Feng G. Neurobiology of obsessive-compulsive disorder: insights into neural circuitry dysfunction through mouse genetics. Curr Opin Neurobiol 2011;21:842-8.

12. Krain AL, Wilson AM, Arbuckle R, et al. Distinct neural mechanisms of risk and ambiguity: a meta-analysis of decision-making. Neuroimage 2006;32:477-84.

13. Paulus MP. Decision-making dysfunctions in psychiatry - altered homeostatic processing? Science 2007;318:602-6.

14. Kerr A, Zelazo PD. Development of "hot" executive function: the children's gambling task. Brain Cogn 2004;55:148-57.

15. Goodman WK, Price LH, Rasmussen SA, et al. The Yale-Brown Obsessive Compulsive Scale: I. Development, use, and reliability. Arch Gen Psychiatry 1989;46:1006-11.

16. Maier W, Buller R, Philipp M et al. The Hamilton Anxiety Scale: reliability, validity and sensitivity to change in anxiety and depressive disorders. J Affect Disord 1988;14:61-8.

17. Macoveanu J, Rowe JB, Hornboll B, et al. Playing it safe but losing anyway - serotonergic signaling of aversive outcomes in dorsomedial prefrontal cortex in the context of risk-aversion. Eur Neuropsychopharmacol 2013;23:919-30.

18. Liu X, Hairston J, Schrier M, et al. Common and distinct networks underlying reward valence and processing stages: a meta-analysis of functional neuroimaging studies. Neurosci Biobehav Rev 2011;35:1219-36.

19. Tzourio-Mazoyer N, Landeau B, Papathanassiou D, et al. Automated anatomical labeling of activations in SPM using a macroscopic anatomical parcellation of the MNI MRI single-subject brain. Neuroimage 2002;15:273-89.

20. Giuliani NR, Pfeifer JH. Age-related changes in reappraisal of appetitive cravings during adolescence. Neuroimage 2015;108:173-81.

21. Guassi Moreira JF, Telzer EH. Mother still knows best: maternal influence uniquely modulates adolescent reward sensitivity during risk taking. Dev Sci 2018;21:e12484.

22. Van Hoorn J, McCormick EM, Rogers CR, et al. Differential effects of parent and peer presence on neural correlates of risk taking in adolescence. Soc Cogn Affect Neurosci 2018;13:945-55.

23. Quintana DS, Williams DR. Bayesian alternatives for common null-hypothesis significance tests in psychiatry: a non-technical guide using JASP. BMC Psychiatry 2018;18:178.

24. Jeffreys H. The theory of probability. Oxford, United Kingdom: Oxford University Press; 1998.

25. Kennerley SW, Walton ME, Behrens TE, et al. Optimal decision making and the anterior cingulate cortex. Nat Neurosci 2006;9:940. 
26. Fitzgerald KD, et al. Error-related hyperactivity of the anterior cingulate cortex in obsessive-compulsive disorder. Biol Psychiatry 2005;57:287-94.

27. Norman LJ, Taylor SF, Liu Y, et al. Error processing and inhibitory control in obsessive-compulsive disorder: a meta-analysis using statistical parametric maps. Biol Psychiatry 2019;85:713-25.

28. Liemburg EJ, Swart M, Bruggeman R, et al. Altered resting state connectivity of the default mode network in alexithymia. Soc Cogn Affect Neurosci 2012;7:660-6.

29. Odriozola P, Uddin LQ, Lynch CJ, et al. Insula response and connectivity during social and non-social attention in children with autism. Soc Cogn Affect Neurosci 2015;11:433-44.

30. Addicott MA, Sweitzer MM, Froeliger B, et al. Increased functional connectivity in an insula-based network is associated with improved smoking cessation outcomes. Neuropsychopharmacology 2015;40:2648.

31. Shaffer JJ, Johnson CP, Fiedorowicz JG, et al. Impaired sensory processing measured by functional MRI in bipolar disorder manic and depressed mood states. Brain Imaging Behav 2018;12:837-47.

32. Mitterschiffthaler MT, et al. Neural response to pleasant stimuli in anhedonia: an fMRI study. Neuroreport 2003;14:177-82.

33. Critchley HD, Kumari V, Malhi GS, et al. Neural activity relating to generation and representation of galvanic skin conductance responses: a functional magnetic resonance imaging study. J Neurosci 2000;20:3033-40.

34. Moreira P, Marques P, Soriano-Mas C, et al. The neural correlates of obsessive-compulsive disorder: a multimodal perspective. Transl Psychiatry 2017;7:e1224.

35. Admon R, Bleich-Cohen M, Weizmant R, et al. Functional and structural neural indices of risk aversion in obsessive-compulsive disorder (OCD). Psychiatry Res Neuroimaging 2012;203:207-13.

36. Stern ER, Welsh RC, Gonzalez R, et al. Subjective uncertainty and limbic hyperactivation in obsessive-compulsive disorder. Hum Brain Mapp 2013;34:1956-70.

37. Fergus TA, Bardeen JR. Emotion regulation and obsessive-compulsive symptoms: a further examination of associations. J ObsessiveCompulsive Relat Disord 2014;3:243-8.

38. Casado $\mathrm{Y}$, et al. Emotional processing in obsessive-compulsive disorder. J Anxiety Disord 2011;25:1068-71.

39. Thorsen AL, Hagland P, Radua J3, et al. Emotional processing in obsessive-compulsive disorder: a systematic review and metaanalysis of 25 functional neuroimaging studies. Biol Psychiatry Cogn Neurosci Neuroimaging 2018;3:563-71.

40. Ochsner KN, Gross JJ. The cognitive control of emotion. Trends Cogn Sci 2005;9:242-9.

41. de Vries FE, de Wit SJ, Cath DC, et al. Compensatory frontoparietal activity during working memory: an endophenotype of obsessivecompulsive disorder. Biol Psychiatry 2014;76:878-87.

42. Eckert MA, Menon V, Walczak A, et al. At the heart of the ventral attention system: the right anterior insula. Hum Brain Mapp 2009; 30:2530-41.
43. Macoveanu J, Miskowiak K, Kessing LV, et al. Healthy co-twins of patients with affective disorders show reduced risk-related activation of the insula during a monetary gambling task. J Psychiatry Neurosci 2016;41:38-47.

44. Palminteri S, Justo D, Jauffret C, et al. Critical roles for anterior insula and dorsal striatum in punishment-based avoidance learning. Neuron 2012;76:998-1009.

45. Morgado P, Marques F, Ribeiro B, et al. Stress induced risk-aversion is reverted by D2/D3 agonist in the rat. Eur Neuropsychopharmacol 2015;25:1744-52.

46. Chang LJ, Yarkoni T, Khaw MW, et al. Decoding the role of the insula in human cognition: functional parcellation and large-scale reverse inference. Cereb Cortex 2013;23:739-49.

47. Droutman V, Bechara A, Read SJ. Roles of the different subregions of the insular cortex in various phases of the decisionmaking process. Front Behav Neurosci 2015;9:309.

48. Christopoulos GI, Tobler PN, Bossaerts P, et al. Neural correlates of value, risk, and risk aversion contributing to decision making under risk. J Neurosci 2009;29:12574-83.

49. Tom SM, Fox CR, Trepel C, et al. The neural basis of loss aversion in decision-making under risk. Science 2007;315:515-8.

50. Hollerman JR, Tremblay L, Schultz W. Involvement of basal ganglia and orbitofrontal cortex in goal-directed behavior. Prog Brain Res 2000;126:193-215.

51. Hauser TU, Iannaccone R, Dolan RJ, et al. Increased fronto-striatal reward prediction errors moderate decision making in obsessivecompulsive disorder. Psychol Med 2017;47:1246-58.

52. Remijnse PL, Nielen MM, van Balkom AJ, et al. Reduced orbitofrontal-striatal activity on a reversal learning task in obsessive-compulsive disorder. Arch Gen Psychiatry 2006;63: 1225-36.

53. Cardoso Cde O, Branco LD, Cotrena C, et al. The impact of frontal and cerebellar lesions on decision making: evidence from the Iowa Gambling Task. Front Neurosci 2014;8:61.

54. Clausi S, Coricelli G, Pisotta I, et al. Cerebellar damage impairs the self-rating of regret feeling in a gambling task. Front Behav Neurosci 2015;9:113.

55. Norman LJ, Carlisi CO, Christakou A, et al. Frontostriatal dysfunction during decision making in attention-deficit/ hyperactivity disorder and obsessive-compulsive disorder. Biol Psychiatry 2018;3:694-703.

56. Lawrence NS, Wooderson S, Mataix-Cols D, et al. Decision making and set shifting impairments are associated with distinct symptom dimensions in obsessive-compulsive disorder. Neuropsychology 2006; 20:409-19.

57. Eklund A, Nichols TE, Knutsson H. Cluster failure: why fMRI inferences for spatial extent have inflated false-positive rates. Proc Natl Acad Sci U S A 2016;113:7900-5.

58. Yeung AW. An updated survey on statistical thresholding and sample size of fMRI studies. Front Hum Neurosci 2018;12:16. 